\title{
Radio detection of cosmic-ray air showers with the OVRO-LWA: status and future plans.
}

\section{Andrés Romero-Wolf*}

Jet Propulsion Laboratory, California Institute of Technology, Pasadena, CA, USA

E-mail: Andrew.Romero-Wolfejpl.nasa.gov

Ryan Monroe $^{1}$, Gregg Hallinan ${ }^{1}$, Washington Carvalho $\mathrm{Jr}^{2,1}$, Kathryn Plant $^{1}$, Konstantin Belov ${ }^{3}$, Anna Nelles ${ }^{4,5}$, Michael Eastwood ${ }^{1}$, Marin Anderson ${ }^{1}$, Larry D'Addario', Jonathon Kocz' ${ }^{1}$, David Woody ${ }^{6}$,

${ }^{1}$ Department of Astronomy, California Institute of Technology, Pasadena, CA, USA;

${ }^{2}$ Departamento de Física, Universidade de São Paulo, São Paulo, Brazil; ${ }^{3}$ Jet Propulsion

Laboratory, California Institute of Technology; ${ }^{4}$ ECAP, FAU Erlangen, Germany; ${ }^{5}$ DESY,

Platanenalle 6, Zeuthen, Germany; ${ }^{6}$ California Institute of Technology, Owens Valley Radio

Observatory, Big Pine, CA, USA;

\begin{abstract}
We present the successful radio frequency $(30-80 \mathrm{MHz})$ self-triggered detection and identification of 10 high energy cosmic rays with Caltech's Owens Valley Radio Observatory Long Wavelength Array (OVRO-LWA). The core of the OVRO-LWA consists of 256 dual-polarization antennas that are approximately evenly spaced in a $200 \mathrm{~m}$ diameter disk with several outliers at longer distances. Based on this success we present future plans for an array covering a $2.5 \mathrm{~km}$ diameter area for measuring the flux and composition of cosmic rays in the $3 \times 10^{16} \mathrm{eV}-10^{18} \mathrm{eV}$ energy band.
\end{abstract}

36th International Cosmic Ray Conference -ICRC2019-

July 24th - August 1st, 2019

Madison, WI, U.S.A.

${ }^{*}$ Speaker. 


\section{Introduction}

The break in the cosmic ray spectrum in the energy range between $10^{17}-10^{18} \mathrm{eV}$, commonly referred to as the second knee, is hypothesized to be the transition between galactic and extragalactic cosmic rays (see [1] for a recent review). Measurements using the KASCADE-Grande array indicate that the composition transitions from heavy to light with increasing energy [2,3]. However, the relation between the shift in composition and the all-particle spectrum is not well understood $[4,5]$.

Radio detection of air showers has become a promising technique for measuring the composition (via estimates of shower maximum $X_{\max }$ ) and energy [6] of cosmic ray air showers. Measurements with LOFAR [7] have demonstrated accurate estimates of $X_{\max }\left(\sim 16 \mathrm{~g} / \mathrm{cm}^{2}\right.$ uncertainty on the mean) in the energy range between $10^{17}-10^{17.5} \mathrm{eV}$. A high statistical sample of events observed with radio arrays has the potential to contribute new measurements of the composition and energy spectrum with a technique that is independent of the KASCADE-Grande [2, 3], Tunka [4] and Telescope Array [5] results. We summarize the demonstration of self-triggered radio detection of cosmic rays with the Owens Valley Radio Observatory Long Wavelength Array (OVRO-LWA) to be presented in [9]. An upgrade of this observatory is currently under construction that will run continuously and provide a high statistical sample of cosmic ray air showers in the $3 \times 10^{16}-10^{18} \mathrm{eV}$.

\section{The Owens Valley Radio Observatory Long Wavelength Array}

The OVRO-LWA was originally designed as a fully cross-correlating interferometer to be used for astronomical purposes, transient science [10], and cosmology [11]. The array consists of 288 dual-polarization antennas (of which only 256 are in active use) distributed across a 1.53 $\mathrm{km}$-diameter region. A $200 \mathrm{~m}$ diameter core contains 251 of these antennas and was the focus of the demonstration effort. The distribution of these antennas is shown in Figure 1. Each antenna sees nearly the entire sky, with a $3 \mathrm{~dB}$ point of approximately $40^{\circ}$ elevation angle. RF signals are baseband-sampled at $F_{S}=196.6 \mathrm{MSPS}$, for a Nyquist frequency of $98.3 \mathrm{MHz}$. This is performed with 8-bit ADC16x250-8 Analog to Digital Converter (ADC) boards. Full technical details are available at [12]. For a description of the planned upgrades, see [13].

\section{Trigger Design}

The OVRO-LWA antennas are routed to an array of FPGA-based processors that is used to produce Fast Fourier Transforms of the data in real-time (F-engine) that are then transferred to GPU to cross-correlation (X-engine). The trigger was implemented by modifying the firmware in the FPGAs normally used as an F-engine. Each of 16 FPGAs processes the data from 16 antennas (in both polarizations) clustered in north-south strips in the core of the array (see right panel of Figure 1).

At the single antenna polarization level, the trigger takes the continuously digitized data and applies a 40-tap filter in the 30-80 MHz band. The filtered signals are squared and smoothed by taking a running average of 4 contiguous samples. If the smoothed power exceeds a threshold set to keep the global trigger rate below $40 \mathrm{~Hz}(10-40 \mathrm{~Hz}$ typical), a $712 \mathrm{~ns}$ coincidence window 


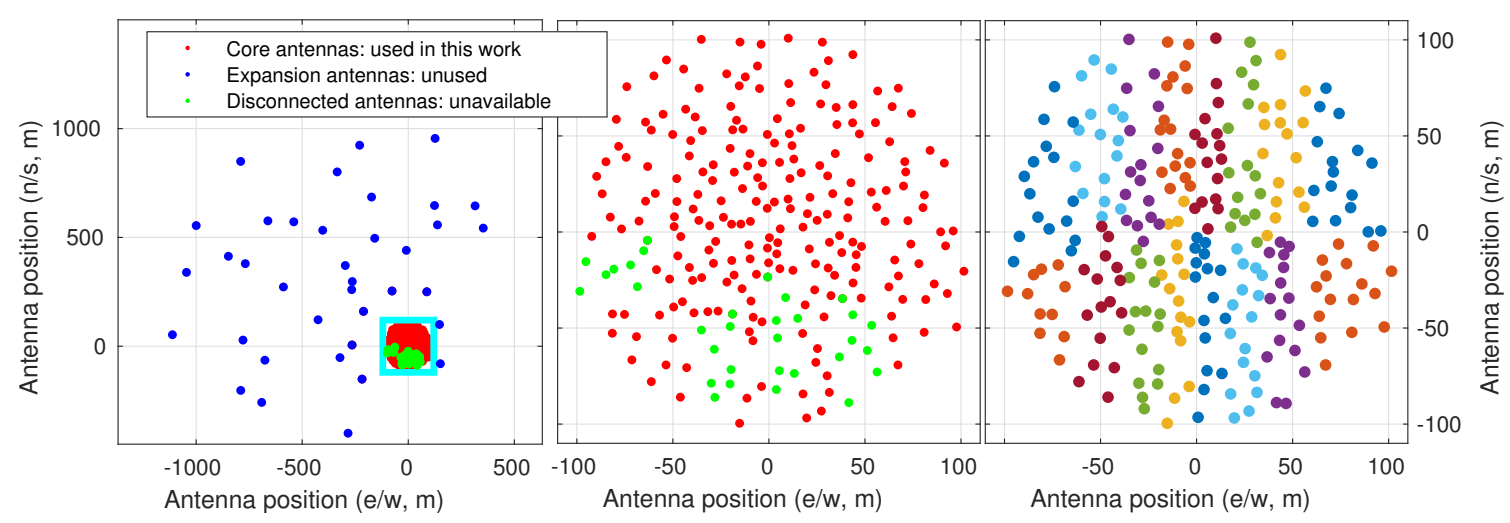

Figure 1: Left: a summary of the 288 OVRO-LWA antennas currently installed. Red antennas were used in this work. Blue antennas could be used in this work, but were excluded in favor of the dense core. Green antennas are physically present and available in an alternative array configuration, but are not used in this work. Center: the same contents, zoomed on the cyan box. The 251 "core" antennas, randomly distributed across a diameter of $200 \mathrm{~m}$, are shown. Right: The same antennas as displayed in the center figure. Each adjacent cluster of antennas which share the same color are assigned to the same FPGA, and are therefore grouped together for the purposes of initial triggering.

(corresponding to the $200 \mathrm{~m}$ diameter of the array) is activated. For each FPGA cluster, at least 12 of 16 antennas for a given polarization are required to trigger within the coincidence window. If an FPGA cluster meets these conditions, the array is triggered with $2.6 \mathrm{~ms}$ dead time and all channels are saved with a 4096 sample window for analysis.

Radio frequency interference (RFI) was found to dominate the trigger event rate (Figure 2). This is predominantly due to nearby arcing power lines. An RFI blocking strategy was developed that used directional rejection and blocking repeating triggers. Although RFI blocking was a promising approach (Figure 2), the best run used in this report was taken during a period of rain, which suppresses the arcing. These RFI mitigation techniques are being developed for the upgrade, and, more importantly, an effort was funded by Caltech/JPL to identify arcing power lines and report them to the Los Angeles Department of Water and Power for repair.

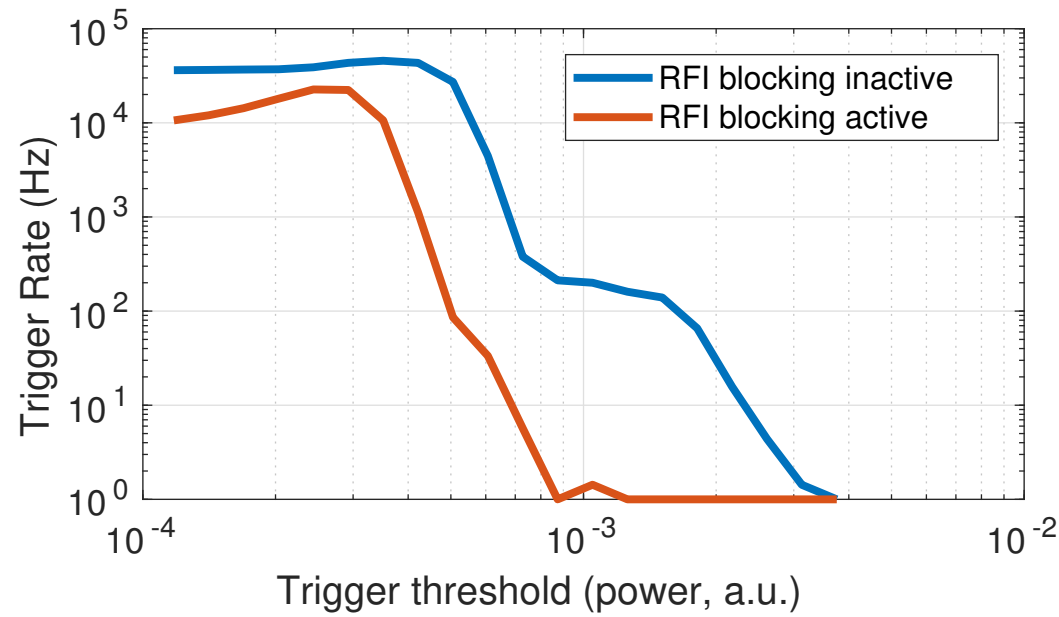

Figure 2: Event rates both with and without on-chip filtering applied. Each data point was collected at a distinct time in a time-variable RFI environment, which limits interpretability of the data. Because each data point is taken at a distinct time, it is not possible to differentiate between time variable RFI and random thermal noise using the data from this plot. 


\section{Background Rejection}

During the 40 hour run $\sim 6 \mathrm{M}$ events triggered the array. There were several quality cuts applied to the data. The peak power values of smooth data are identified and a cut is applied requiring an SNR $>5$. The time of arrival is used and both a plane wave and spherical wavefront (e.g. nearby source) fits are applied. If the events are not consistent with a plane wave they are cut. In addition, some events had incomplete readouts resulting in only a small number of antennas with data ( $\sim 22 \%$ of events). A total of $473 \mathrm{~K}$ events survive these basic quality cuts. The upper left panel of Figure 3 shows the spatio-temporal distribution of the surviving events. Events that come from directions that produce significant amounts of RFI, such as the cities of Bishop and Big Pine along with other directions, are flagged (colored orange in the upper left panel of Figure 3) and cut out of the dataset.

The remaining events are airplane tracks that manifest themselves as spatio-temporally correlated tracks (upper right panel of Figure 3). To flag these events, a curve fitting algorithm was applied to identify the smooth trajectories produced by airplanes. After these cuts are applied, a subset of events are found to be temporally clustered and cut from the sample (lower left panel of Figure 3). The final set of surviving events (lower right panel of Figure 3) are promoted to further inspection and checked for consistency with cosmic ray air showers.

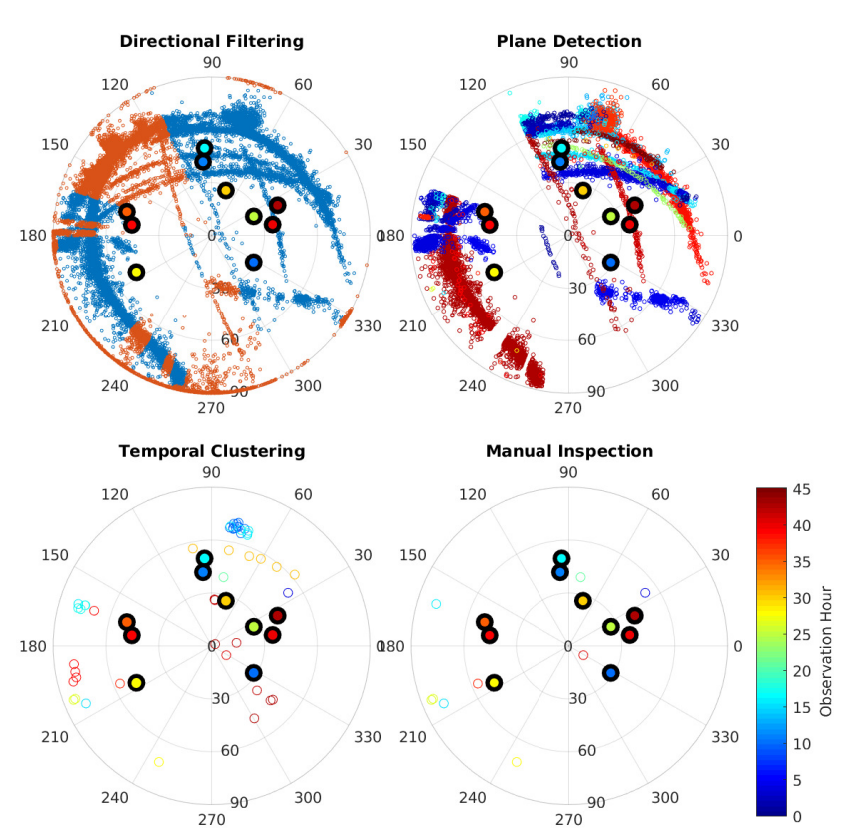

Figure 3: A spatio-temporal representation of the events at different stages of the analysis. Each point represents an event azimuth and zenith angle (North is up). Cosmic ray candidate events are shown with black edges on their markers. Upper left: All 473K events in the dataset which pass instrumental and event power checks. Events marked red are flagged based on azimuth and elevation cuts. Upper right: Colors indicate observation hour of event arrival (see colorbar in bottom right). Airplane tracks manifest themselves as spatio-temporal clustered stripes. Lower left: Events surviving the airplane-fitting routine show temporal clustering, which are further filtered. Lower right: Events which are promoted to final cuts and human inspection.

\section{Cosmic Ray Identification}

The principal identifiers of cosmic ray air showers are the degree-scale beam pattern, polarization correlation with the geomagnetic field, and pulse arrival times [8]. The timing infromation is used earlier in the analysis by requiring the events are consistent with a plane wave. Although cosmic rays are known to deviate from plane waves, over the $200 \mathrm{~m}$ extent of the OVRO-LWA, the deviations are small and do not impact the fit. The beam pattern (see candidate event in Figure 4) is 
approximated by a two-dimensional Gaussian fit and the fraction of power captured in the Gaussian compared to a flat profile is estimated. The polarization is estimated comparing the relative power in each polarization. The uncertainty is $\sim 20 \%$ level lacking more detailed modeling dipole beam patterns, which will be included in future developments.

A scatter plot of the surviving events in polarization residual error vs fractional power captured in Gaussian fit is shown in Figure 5. Cosmic ray candidates are separated from RFI by requiring the residual polarization error is $<20 \%$ and the fractional power captured by Gaussian fit is $>20 \%$.

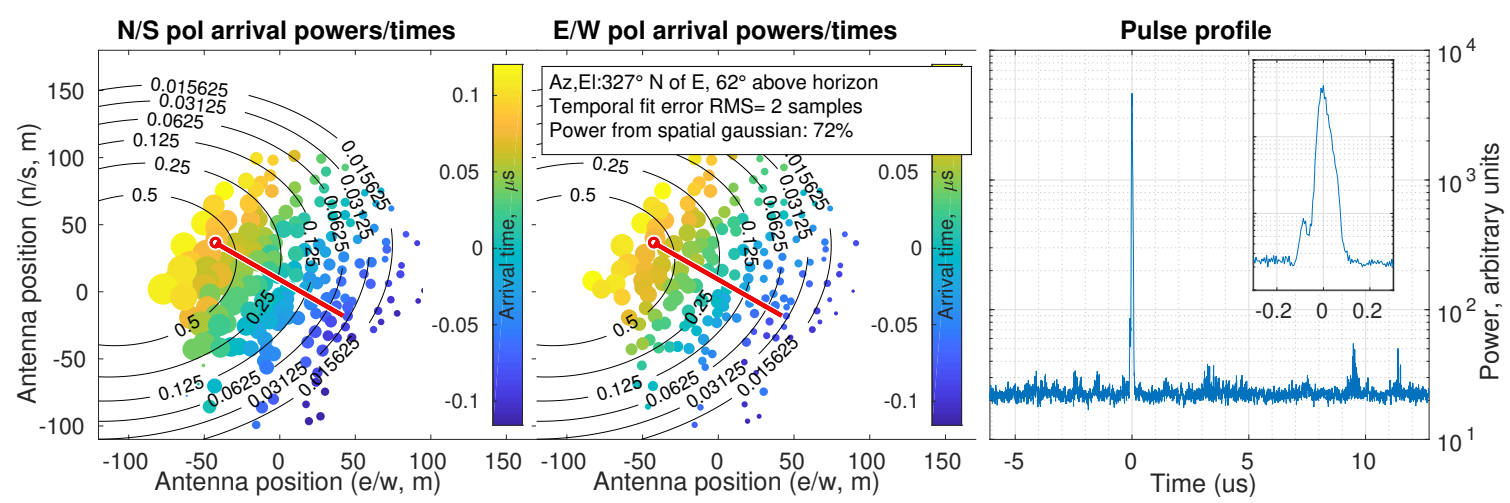

Figure 4: Cosmic ray candidate event. The event has maximum power to the northeast, but arrives from the southwest, which is evidence that this comes from a beamed source (left and center panels). In addition, this event is a band-limited impulse (right panel) and is not spatio-temporally correlated with other events (source information in text inset, but other event information not displayed here). Likewise, the polarization signature is compatible with expected models.

\section{Results}

A detailed simulation was created to test the consistency of the 10 cosmic ray events. A parameterization of ZHAireS beam patterns of [14] along with the geomagnetic field correlations of the polarization are included in the signal simulations. The antenna beam pattern and impedance vs frequency were estimated using a NEC simulation to estimate the effective height. We used a Galactic noise spectrum and compared it to the measured noise spectrum to estimate the average response of the filters. Cosmic rays were sampled in 0.1 dex of energy for the trigger simulation. The threshold amplitude signal to noise ratio (peak voltage $V_{\mathrm{pk}}$ divided by noise rms voltage $V_{\mathrm{rms}}$ ) is $\sim 5.7$ based on inspection of the surviving events.

We included estimates of the quality cuts (QC) based on directional rejection and airplanes which are $\sim 75 \%$ efficient. The fractional power in Gaussian cut (GC) is $\sim 60 \%$ efficient. Most of the losses are due to inclined events which tend to illuminate the $200 \mathrm{~m}$ diameter core of the array uniformly. The combined efficiency of these cuts is $\sim 45 \%$.

The resulting acceptance and expected event rates as a function of energy are shown in Figures 6 and 7, respectively. In Figure 8 we show the total expected number of cosmic ray air showers detected in a 40 hour run as a function of amplitude SNR threshold for the trigger and with the analysis cuts applied. The 10 candidate events detected are consistent with the simulations. The black line shows the number expected to trigger the array. The uncertainties shown are statistical only. 


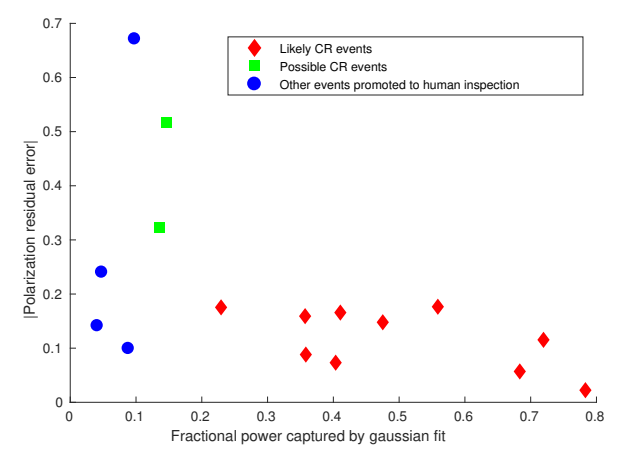

Figure 5: Final cuts on the expected cosmic ray polarization and radiation footprint for events which pass all subsequent criteria. See text for details.

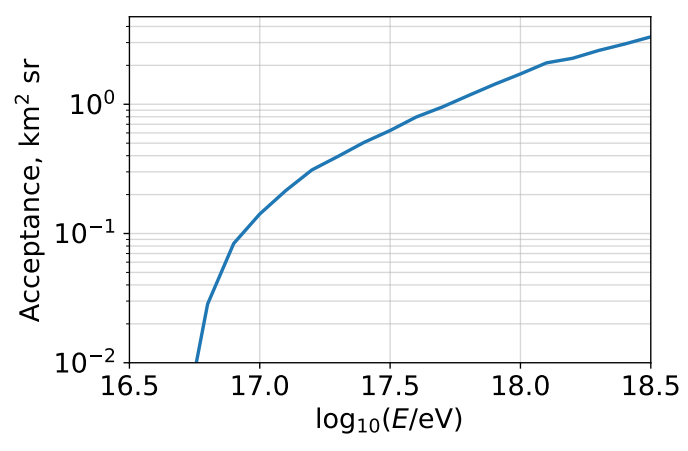

Figure 6: Acceptance as a function of energy for cosmic ray events that trigger the OVRO-LWA detector $\left(\mathrm{SNR}_{\mathrm{a}}>5.7\right)$ including the analysis efficiency.

The red line shows the number of triggered events that pass the quality cuts (labelled QC) and the red line includes both the QC and power in Gaussian cut (labelled GC). See text for details on the cuts. The light yellow region is the range of trigger thresholds based on the amplitude SNR threshold of 5.7 with an uncertainty corresponding to the noise voltage fluctuations $V_{\text {rms. }}$. The green region indicates the 10 events observed with a Poisson uncertainty range. As an additional consistency check, we estimated the distribution of arrival zenith angle directions of cosmic rays from our simulation, including the QC and GC analysis efficiencies, and compared it to the data showing they are consistent (Figure 9).

As a final check, we estimated the probability that the cosmic ray candidate events are false positives. We took a sample of impulsive events and estimated the following probabilities based on the distribution of the data: the fractional power in Gaussian fit below a given value, the polarization residual error above a given value, the nearest event in time, and the angular distance to the nearest event in time. We then took the product of the probabilities assigned to each event with the resulting distribution shown in Figure 10. Events with high probabilities are background like and small probabilities deviate from the main distribution. The cosmic ray candidate are all outliers from the main distribution. One impulsive event that did not meet the criteria for a cosmic ray candidate (near a product of probabilities of $\gtrsim 10^{-8}$ ) was identified. Upon inspection, this event overlapped with a flight path although not at the time where the flight track was active. This shows our cuts were likely too conservative and that this event could be an additional cosmic ray candidate.

\section{Future Developments}

The successful demonstration of self-triggered radio detection of air showers has led to plans to have a commensal cosmic ray trigger and data reduction pipeline that runs continuously along with other OVRO-LWA observations which include radio detection of astronomical radio transient, cosmological observations of the redshifted $21 \mathrm{~cm}$ hydrogen line, solar radio bursts, and Jovian bursts including characterization of icy moons via reflected emissions. Along with the implementation of this trigger, the array is being expanded from 288 antennas to 352 filling in the $2.5 \mathrm{~km}$ region beyond the core of the array. These antennas will be used in future detection improving the Gaussian 

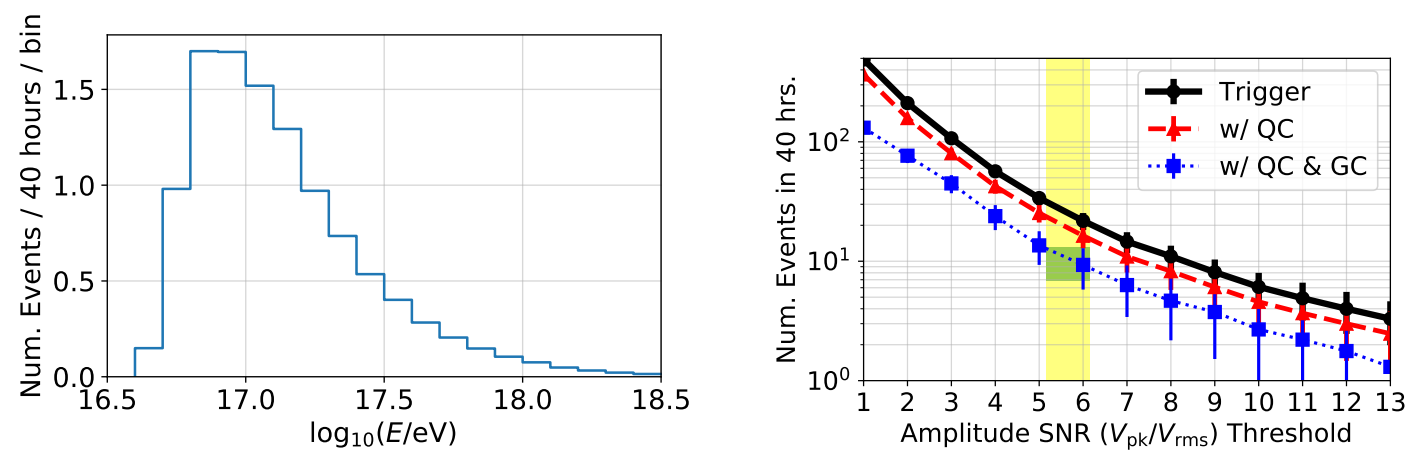

Figure 7: Number of events function of energy for cosmic ray events that trigger the OVRO-LWA detector (SNR > 5.7) including the analysis efficiency.

Figure 8: Number of expected events in a 40 hour run as a function of peak amplitude trigger threshold and analysis cuts. See text for details.
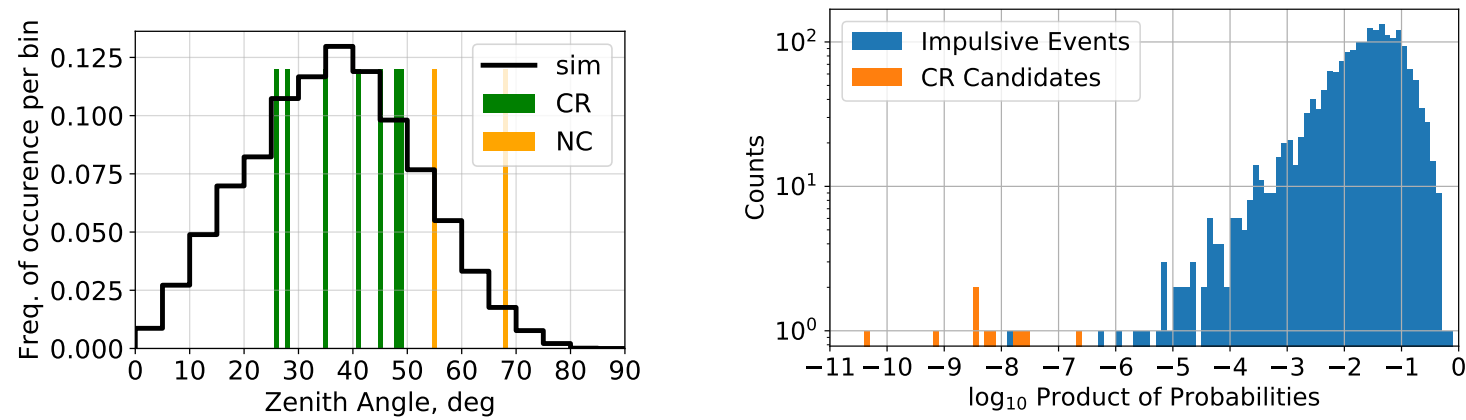

Figure 9: Frequency of occurrence of cosmic rays Figure 10: Histogram of the combined probabilities dethat trigger the OVRO-LWA detector $\left(\mathrm{SNR}_{\mathrm{a}}>5.7\right)$ scribed in the text. The non-CR candidate event was and analysis cuts. The zenith angles of cosmic ray rejected due to clustering with a known flight path event candidates (CR) are shown in green bars with the though it was temporally isolated from other impulsive "no call" (NC) events (a.k.a. "possible CR events" transients.

in Figure 5) shown in orange bars.

quality cut by virtue of being able to sample a larger portion of the air shower radio emission beam. As part of the upgrade, the antenna polarization will be calibrated to $3 \%$ uncertainty with a goal of reaching $1 \%$, driven by the companion science objectives of the array. This has the potential to significantly improve RFI rejection efficiency. In addition to repairing nearby arcing power lines, the trigger design for the upgrade builds on this initial study to improve RFI rejection at the trigger level in the FPGA using directional and repeated burst rejection. It is expected that this new system will produce $>2,000$ events per year. For details on the upgraded trigger system design see [13].

The upgraded OVRO-LWA will produce a high statistical sample of events with $X_{\max }$ resolution comparable to and possibly better than LOFAR with energy resolution $<20 \%$ (for details see [15]). A large statistical sample will improve measurements of the all-particle spectrum along with composition estimates in the $3 \times 10^{16}-10^{18} \mathrm{eV}$ energy band. In addition, the array will have sub-degree resolution to enable anisotropy studies and searches for neutron sources similar to the ones performed by Auger [16] but in a lower energy band. With the expected $X_{\max }$ resolution, we 
are also currently investigating flux limits that may be placed on gamma ray sources at energies $>1 \mathrm{PeV}$.

Acknowledgements: Part of this work was carried out at the Jet Propulsion Laboratory, California Institute of Technology, under a contract with the National Aeronautics and Space Administration. A. R.-W. and G. H. thank the Caltech/JPL President's and Director's Research and Development Fund. W.C. thanks grants \#2015/15735-1 and \#2018/18876-3, São Paulo Research Foundation (FAPESP). This material is based in part upon work supported by the National Science Foundation under Grant AST-1654815 and AST-1212226. The OVRO-LWA project was initiated through the kind donation of Deborah Castleman and Harold Rosen. Copyright 2019. All rights reserved.

\section{References}

[1] F. G. Schröder, et al., Bulletin of the AAS, 51(3) 133, (2019).

[2] KASCADE-Grande Collaboration, W. D. Apel et al., Phys. Rev. Lett. 107 (2011) 171104.

[3] KASCADE-Grande Collaboration, W. D. Apel et al., Phys. Rev. D87 (2013) 081101.

[4] Tunka-133 Collaboration, V. Prosin et al., Nucl.Instrum. Meth. A756 (2014) 94.

[5] Telescope Array Collaboration, R. Abbasi et al., Astrophys. J. 865 (2018) 74.

[6] T. Heuge, Physics Reports 620 (2016) 1.

[7] S. Buitink et al., Nature, 531 (2016) 70.

[8] F. Schröder et al., Progress in Particle and Nuclear Physics, 93 (2017) 1.

[9] R. Monroe et al., submitted for publication to Nucl. Instrum. Meth. A.

[10] M. M. Anderson et al., ApJ, 864 (2018) 22.

[11] M. Eastwood et al., ApJ, 156 (2018) 32.

[12] J. Kocz et al., JAI, 4 (2015) 155003.

[13] K. Plant et al., PoS(ICRC2019)391 (2019).

[14] J. Alvarez-Muñiz et al., Astroparticle Physics, 59 (2014) 29.

[15] W. Carvalho Jr. et al., PoS(ICRC2019)211 (2019).

[16] Pierre Auger Collaboration, P. Abreu et al., ApJ, 760 (2012) 148. 